months in children with intractable IS and 2-4 weeks in those with new-onset IS. Mean age at diet onset was 1.2 years, and diet duration was a mean of 1.3 years. An initial 3.1:1 or 3.5:1 ratio of fat to carbohydrate and protein was used in $68(65 \%)$ patients; a $4: 1$ ratio was started in 16 of older age patients. All patients fasted prior to diet initiation. Parents kept patient records of daily seizure frequency, weekly weight, and biweekly urine ketones. Intent-to-treat analysis showed $>50 \%$ control of spasms in $64 \%$ at 6 months and $77 \%$ after 1-2 years. Complete control for at least 6 months within a median of 2.4 months was achieved in 38 (37\%). Improvement in development occurred in $62 \%$, EEG improvement in $35 \%$, and reduction in current anticonvulsants in $29 \%$. Adverse effects developed in $33 \%$, with diminished linear growth in $6 \%$. Older age at onset of IS and fewer prior anticonvulsants were associated with $>90 \%$ spasm reduction at 6 months. In this prospective trial, two thirds of patients with IS treated with the ketogenic diet were benefited, and the diet is strongly recommended for patients who have failed to respond to corticosteroids and vigabatrin. (Hong AM, Turner Z, Hamdy RF, Kossof EH. Infantile spasms treated with the ketogenic diet: Prospective single-center experience in 104 consecutive infants. Epilepsia Aug 2010;51(8):1403-1407). (Respond: Eric H Kossof MD, The Johns Hopkins Hospital, Baltimore, MD 21287. E-mail: ekossof@jhmi.edu).

COMMENT. Nordli DR Jr and associates at Children's Memorial Hospital, Chicago, have previously demonstrated the efficacy and safety of the ketogenic diet in the treatment of 32 infants with intractable seizures, particularly for infantile spasms/myoclonic seizures. (Nordli DR Jr et al. Pediatrics 2001;108(1):129-133). Fifty five percent of infants had $>50 \%$ reduction in seizure frequency ( $19 \%$ were seizure-free). Improvements in behavior, attention/alertness, activity level and socialization were also reported. The diet was well tolerated, and $96 \%$ maintained appropriate growth. Adverse events were all reversible.

\title{
EFFECT OF PRICE ON USE OF ACTH FOR INFANTILE SPASMS
}

The effect of the 14-fold price increase on Aug 27, 2007 of ACTH treatment of infantile spasms in the US was evaluated at University of Colorado, Denver, in 97 patients treated 2007-2009. Before the price increase, patients were more likely to be treated with ACTH as first choice, and were hospitalized for 2.2+/-0.5 SD days for initiation. After price increase, oral AEDs were a more likely first treatment $(\mathrm{P}<0.002)$, and those selected for ACTH were hospitalized significantly longer (5.1+/-0.6 days, SD $\mathrm{P}<0.001)$. ACTH is considered the most effective treatment for infantile spasms and before $2007,88 \%$ of child neurologists in the US used ACTH as initial therapy. In late 2009 , vigabatrin became the only approved drug for infantile spasms in the US. UK studies in 2004 and 2005 found that hormonal treatment more often stopped spasms and improved outcomes than vigabatrin. Since the studies, treatment practices in the UK changed in 2008 in favor of steroids. Whereas the natural form of ACTH is used in the US, accounting in part for the price increase, a synthetic form is used elsewhere. Pricing and availability are influencing the choice of treatment and hospitalization for infantile spasms. (Wray CD, Benke TA. Effect of price increase of adrenocorticotropic hormone on treatment practices of infantile spasms. Pediatr Neurol Sept 2010;43:163-166). (Respond: E-mail: carterwraymd@gmail.com). 